\title{
INFECCIÓN POR HONGOS FILAMENTOSOS EN PACIENTES QUEMADOS: ESTUDIO RETROSPECTIVO.
}

\author{
FILAMENTOUS FUNGAL INFECTION IN BURNED PATIENTS: RETROSPECTIVE STUDY.
}

\author{
María Florencia Spesso ${ }^{1,3}$, Susana Aiassa ${ }^{1}$, Alicia Garutti ${ }^{1}$, Mariel Carballo ${ }^{2}$, Gladys Dotto ${ }^{1}$
}

\section{Resumen:}

Introducción: Las infecciones son las complicaciones más frecuentes en los pacientes quemados. Los hongos filamentosos ocupan un lugar incierto dentro de las estadísticas nosocomiales; existen escasos datos sobre los agentes causales y el desenlace de los que las padecen. El objetivo fue evaluar prevalencia de la infección por hongos filamentosos (IHF), en pacientes internados en la unidad terapia intensiva de quemados (UTIQ), revisar algunas características clínicas, epidemiológicas, microbiológicas y evolución de estos pacientes; conocer la frecuencia y distribución de las especies fúngicas aisladas. Materiales y métodos: estudio retrospectivo y descriptivo. Se incluyeron todos los individuos que ingresaron a la terapia intensiva de pacientes quemados (2012-2015), con cultivo positivo para hongos filamentosos en biopsias de piel y escaras. Resultados: un total de 168 pacientes fueron ingresados a la UTIQ en el período agosto 2012 - agosto 2015. El 17\% (29/168) presentaron cultivos positivos para hongos filamentosos, con 93\% de exámenes microscópicos positivos. Se obtuvieron los siguiente géneros fúngicos: Aspergillus spp (24\%), Fusarium spp (14\%), Mucor spp (3\%) y diversos géneros de hongos negros (58\%). El $90 \%$ fueron de los infectados fueron grandes quemados. El $24 \%$ de los pacientes con IHF falleció y Fusarium spp fue hallado en el $50 \%$ de los casos. La causa de la muerte fue shock cardiogénico irreversible con falla multiorgánica. Conclusiones: La infección por hongos filamentosos se presentó en el 17\% de los pacientes quemados. Los principales hongos causantes de infección fúngica fueron dematiáceos. La mortalidad entre los pacientes fue del $24 \%$, siendo Fusarium el hongo hallado en el mayor número de muertes.

Palabras clave: hongos; unidades de quemados; micosis.

\section{Abstract:}

Introduction: Infections are the most frequent complications in burn patients. Filamentous fungi have an uncertain place within the statistics, since in our media data regarding their prevalence, causal agents and outcome of those who suffer them remain scarce. The aim of this study was to evaluate the prevalence of fungal infection by filamentous fungi (IHF) in patients hospitalized in burn intensive care unit (BICU), and to review clinical, epidemiological, microbiological and evolutionary characteristics of these patients and to know the frequency and distribution of the isolated fungi. Materials and methods: Retrospective and descriptive study. It includes all individuals admitted in burns intensive care unit (2012-2015), with positive culture for filamentous fungi in skin biopsies and bedsores. Results: A total of 168 patients were admitted in the BICU in a period of 3 years. 90\% were major burned and 17\% of them developed IHF (29/168). Aspergillus spp (24\%), Fusarium sp (14\%), Mucor spp (3\%) and various black fungus genera (58\%) were the main genera found in cultives. About $24 \%$ of the patients with IHF died and Fusarium spp was found in $50 \%$ of the cases. The cause of death was irreversible cardiogenic shock with multiorgan failure. Conclusions: Filamentous fungal infection was present in $17 \%$ of burned patients. The main isolatte fungi in samples were dematiaceous mould. Mortality among patients was $24 \%$, with Fusarium being the fungus found in the highest number of deaths (50\%).

Keywords: fungi; burn units; mycoses.

1 División Bacteriología, Área Microbiología. Hospital Córdoba. Córdoba. Argentina.

2 Departamento Laboratorio Cátedra de Dermatología. Hospital Nacional de Clínicas. Facultad de Ciencias Médicas .Universidad Nacional de Córdoba. Argentina

3 Email de contacto: florenciaspesso@yahoo.com.ar 


\section{Introducción}

La incidencia de las infecciones fúngicas en los pacientes quemados varía de unas unidades a otras ${ }^{1-6}$. La respuesta inmune innata y adaptativa se encuentra severamente disminuida en las personas con quemaduras extensas, haciéndolos vulnerables a infecciones oportunistas durante su estadía en la unidad de cuidados intensivos ${ }^{7}$. Los avances en el manejo clínico y terapéutico de estas lesiones ha mejorado la sobrevida de los pacientes, sin embargo, las infecciones continúan siendo su mayor causa de morbimortalidad. Alrededor del $70 \%$ de los pacientes con quemaduras severas fallecen por septicemia vinculada a la infección de sus heridas ${ }^{8}$. Los procedimientos de control de infecciones se han enfocado en el tratamiento de las bacterias y estas estrategias han resultado en su disminución, sin embargo, las causadas por hongos permanecen relativamente estables ${ }^{9}$. La ruptura extensa de las barreras anatómicas, así como el uso de antibióticos de amplio espectro y la internación prolongada son factores que predisponen para su aparición. Existe un predominio de Candida spp. en los pacientes quemados, sin embargo, los hongos filamentosos pueden colonizar las heridas y causar enfermedad ${ }^{6}$.

La mayoría de estos hongos filamentosos son saprofíticos. Su distribución es universal y su presencia depende de las condiciones climáticas (temperatura y humedad) y la disposición de nutrientes para su crecimiento $^{10}$.

Las especies involucradas y la prevalencia de la infección fúngica por hongos filamentosos varían de acuerdo a cada centro de salud.

Es fundamental conocer la epidemiología de cada nosocomio así como los factores de riesgo asociados a su aparición ${ }^{8,9}$, prevenir la infección por estos microrganismos y establecer medidas terapéuticas empíricas adecuadas para su tratamiento.

En Argentina existen escasos estudios sobre estas infecciones en pacientes quemados, todos ellos en población pediátrica ${ }^{5,11}$. En nuestra ciudad, no contamos con datos epidemiológicos que nos permitan conocer ninguno de los aspectos anteriormente mencionados.

El objetivo de nuestro trabajo es evaluar la prevalencia de la infección fúngica por hongos filamentosos en la unidad de cuidados intensivos de quemados del Hospital Córdoba (HC); revisar las características, factores predisponentes y la evolución de los pacientes que padecen este tipo de infecciones y conocer la epidemiología de las especies más frecuentes para establecer medidas terapéuticas efectivas y de manera precoz.

\section{Materiales y Métodos}

Se realizó un estudio retrospectivo y descriptivo basado en la revisión de historias clínicas de los pacientes ingresados en la terapia intensiva de quemados del HC entre agosto de 2012 y agosto de 2015, con cultivo positivo para hongos filamentosos.

Historias Clínicas: Se registraron datos demográficos de los pacientes: edad y sexo, así también antecedentes patológicos previos, características de las quemaduras: porcentaje de superficie corporal quemada (\%SCQ), grado, causa de las quemaduras; evolución y mortalidad de los pacientes.

También se obtuvieron datos del tiempo transcurrido entre la internación y la aparición de lesiones, el resultado del examen directo de las biopsias, cultivos e identificación de las especies aisladas, número de infecciones bacterianas (respiratorias, sistémicas, urinarias y/o de piel y partes blandas).

Datos microbiológicos: Los materiales (biopsia de piel y escaras) fueron procesados según rutinas microbiológicas estandarizadas (microscopía directa y cultivo).

Las muestras se inocularon en medios de cultivo micológicos y bacteriológicos: agar Sabouraud por (duplicado), agar sangre, agar chocolate y caldo tioglicolato y se incubaron a 25 y $37^{\circ} \mathrm{C}$ durante $4-6$ semanas antes de ser informados como negativos. El género de los hongos filamentosos fue definido a través de las características macroscópicas de las colonias y su micromorfología, en preparados montados con azul de lactofenol y microcultivos en agar papa. En algunos casos, los cultivos fueron derivados al Instituto Malbrán cuando no pudieron ser identificados por los métodos convencionales. 
Análisis Estadístico: Se utilizaron métodos estadísticos univariados para el análisis de los datos cuantitativos y las variables categóricas fueron expresadas como valores y porcentajes. Para corroborar la independencia de las variables, se empleó el test estadístico Chi Cuadrado $(p<0.05)$, utilizando el programa online de acceso gratuito de la Social Science Statics (http://www.socscistatistics.com/.). Se calculó el índice de Charlson en función de la edad y la comorbilidad del paciente utilizando la herramienta online de la Sociedad Andaluza de Medicina Intensiva (http://www.samiuc.es/).

Los datos de las historias clínicas se obtuvieron siguiendo los protocolos establecidos por el Comité de Capacitación y Docencia y el Comité de Ética en investigación del Hospital Córdoba, Argentina.

\section{Resultados}

Un total de 168 pacientes fueron ingresados a la UTIQ en el período agosto 2012 - agosto 2015, de los cuales, 17\% (29/168) presentaron cultivos positivos para hongos filamentosos. Los mismos se obtuvieron de muestras de biopsias de piel $(n=36)$ y escaras $(n=2)$ tomadas de diferentes localizaciones anatómicas. La mayoría de los internados no presentaba comorbilidades importantes al momento del ingreso a terapia: diabetes $(n=1)$, tumor sólido sin metástasis $(n=1)$, epilepsia $(n=1)$, obesidad $(n=1)$ y depresión $(n=3)$. El Índice Charlson para estos pacientes fue entre 0 y 2 puntos, por lo que su esperanza de vida a los diez años fue mayor al $90 \%$ en todos los casos.

De los 29 pacientes, 8 debieron ser excluidos del análisis estadístico por no tener datos suficientes en sus historias clínicas, por lo que el $n$ de la muestra se redujo a 21 individuos $(n=21)$.

En total $n=21$ internados fueron incluidos en el estudio: $62 \%$ eran mujeres y $38 \%$ hombres, con una edad promedio de 43 años (Tabla 1). Nuestra institución solo atiende a pacientes mayores de 15 años, por lo que la población pediátrica no está incluida en el estudio.

Tabla 1: Distribución de la muestra según sexo y edad ( $\mathrm{n}=29)$.

\begin{tabular}{|cccccc|}
\hline Sexo & n & Media & DS & Mínimo & Máximo \\
\hline Masculino & 13 & 43,8 & 21 & 15 & 78 \\
Femenino & 16 & 43,4 & 15 & 21 & 74 \\
\hline \multicolumn{5}{c}{ Pie de tabla }
\end{tabular}

Se calculó media, desviación estándar y rango para cada género.

n: número de muestras; DS: desviación estándar.

La causa principal de las quemaduras fueron lesiones térmicas por fuego directo ocasionadas por accidentes domésticos y autoagresión. No se registraron quemaduras químicas ni por radiación; tampoco daño por inhalación.

El promedio de superficie corporal quemada (\%SCQ) fue 31\% (13\%-75\%), y las lesiones fueron de grado $A B$ y $B$ (segundo y tercer grado), lo cual los clasifica como grandes quemados.

Un 95\% presentó entre una y cuatro infecciones bacterianas asociadas a la estadía en la unidad de cuidados intensivos (UTI) además de la infección fúngica, con una media de dos focos para los pacientes que sobrevivieron y tres focos en los óbitos. Se registraron 10 neumonías asociadas al ventilador (47\%),11 bacteriemias (52\%), 13 infecciones de piel y partes blandas (61\%) y 15 infecciones del tracto urinario $(71 \%)$. Se obtuvo desarrollo de 29 hongos filamentosos en total, ya que en algunos casos los pacientes presentaron múltiples lesiones y se envió más de una muestra para cultivo.

El 93\% (27/29) de los exámenes microscópicos fueron positivos, demostrando la presencia de hifas en los tejidos. Se identificaron Aspergillus spp (24\%), Fusarium spp (14\%), Mucor spp (3\%) y diversos géneros de hongos negros $(58 \%)$, de acuerdo a las características macro y micromorfológicas. El tiempo promedio de aparición de las lesiones fue 12 días (1-28). Las infecciones por hongos dematiáceos y Fusarium se registraron entre la 1ra y 4ta semana de internación y Aspergillus spp luego de la segunda semana.

En nuestro centro, el $24 \%(n=7)$ de los pacientes fallecieron debido a las siguientes micosis: $3 / 6$ fusariosis (50\%), 1/7 aspergilosis (14\%) y $3 / 21$ feohifomicosis (14\%). Los géneros hallados en estos individuos fueron: Fusarium spp $(n=3)$, Aspergillus spp $(n=1)$ y distintos géneros de hongos dematiáceos $(n=3)$. El $\%$ SCQ fue del $20 \%$ al $50 \%(x=35 \%)$ sin que hubiera una diferencia estadísticamente significativa respecto 
a los que sobrevivieron. La causa de muerte en todos los casos fue shock cardiogénico irreversible con falla multiorgánica (Tabla 2).

Tabla 2: Características clínicas y microbiológicas de los pacientes quemados con aislamiento de hongos filamentosos en biopsia de piel y escara $(n=29)$.

\begin{tabular}{|c|c|c|c|c|c|}
\hline Paciente & APP & Género & & $\%$ SCQ & Obito \\
\hline 1 & $\mathrm{~N} / \mathrm{R}$ & Bipolaris spp. & Acremonium spp. & $20 \%$ & No \\
\hline 2 & $\mathrm{~N} / \mathrm{R}$ & Mucor spp. & & $43 \%$ & No \\
\hline 3 & $\mathrm{~N} / \mathrm{R}$ & Fusarium spp. & & $27 \%$ & $\mathrm{Si}$ \\
\hline 4 & $\mathrm{~N} / \mathrm{R}$ & Aspergillus spp. & Curvularia spp. & $35 \%$ & $\mathrm{Si}$ \\
\hline 5 & $\mathrm{~N} / \mathrm{R}$ & Hongo filamentoso dematiáceo & & $50 \%$ & $\mathrm{Si}$ \\
\hline 6 & Ca. de mama/Depresión & Fusarium spp. & & $20 \%$ & $\mathrm{Si}$ \\
\hline 7 & $\mathrm{~N} / \mathrm{R}$ & Alternaria spp. & & $40 \%$ & $\mathrm{Si}$ \\
\hline 8 & $\mathrm{~N} / \mathrm{R}$ & Hongo filamentoso dematiáceo & & $36 \%$ & $\mathrm{Si}$ \\
\hline 9 & $\mathrm{~N} / \mathrm{R}$ & Aspergillus spp. & & $40 \%$ & No \\
\hline 10 & $\mathrm{~N} / \mathrm{R}$ & Aureobasidium spp & & $50 \%$ & No \\
\hline 11 & Depresión & Aspergillus spp. & & $20 \%$ & No \\
\hline 12 & $\mathrm{~N} / \mathrm{R}$ & Acremonium spp & & $20 \%$ & No \\
\hline 13 & $\mathrm{~N} / \mathrm{R}$ & Fusarium spp. & & $25 \%$ & No \\
\hline 14 & $\mathrm{~N} / \mathrm{R}$ & Alternaria spp. & & $20 \%$ & No \\
\hline 15 & N/R & Dreschlera spp. & & $25 \%$ & No \\
\hline 16 & $\mathrm{~N} / \mathrm{R}$ & Hongo filamentoso dematiáceo & & $2 \%$ & No \\
\hline 17 & Epilepsia & Alternaria spp. & & $75 \%$ & No \\
\hline 18 & $\mathrm{~N} / \mathrm{R}$ & Fusarium spp. & & $13 \%$ & No \\
\hline 19 & $\mathrm{~N} / \mathrm{R}$ & Alternaria spp. & & $30 \%$ & No \\
\hline 20 & $\mathrm{~N} / \mathrm{R}$ & Curvularia spp & Aspergillus spp. & $28 \%$ & No \\
\hline 21 & $\mathrm{~N} / \mathrm{R}$ & Curvularia spp & & $24 \%$ & No \\
\hline 22 & DBT II/Depresión & Fusarium spp. & & $\mathrm{S} / \mathrm{D}$ & $\mathrm{Si}$ \\
\hline 23 & $\mathrm{~N} / \mathrm{R}$ & Fusarium spp. & & $\mathrm{S} / \mathrm{D}$ & No \\
\hline 24 & $\mathrm{~N} / \mathrm{R}$ & Aureobasidium spp. & & $\mathrm{S} / \mathrm{D}$ & No \\
\hline 25 & $\mathrm{~N} / \mathrm{R}$ & Curvularia spp. & & $\mathrm{S} / \mathrm{D}$ & No \\
\hline 26 & $\mathrm{~N} / \mathrm{R}$ & Curvularia spp. & & $\mathrm{S} / \mathrm{D}$ & No \\
\hline 27 & N/R & Bipolaris spp. & & $\mathrm{S} / \mathrm{D}$ & No \\
\hline 28 & N/R & Curvularia spp. & & $\mathrm{S} / \mathrm{D}$ & No \\
\hline 29 & $\mathrm{~N} / \mathrm{R}$ & Aureobasidium spp. & & S/D & No \\
\hline
\end{tabular}

corporal quemada; N/R: no refiere; n: número de muestras.

El $76 \%$ de los pacientes recibió tratamiento antifúngico (además del debridamiento quirúrgico de las lesiones) luego del diagnóstico y no hubo diferencia significativa en la mortalidad entre los pacientes tratados y no tratados $(p=0,8)$. Las drogas utilizadas fueron Anfotericina B (AMB), Itraconazol (ITRA) y Voriconazol (VORI).

\section{Discusión}

En estos últimos años, se ha registrado un incremento de infección fúngica en quemados a pesar de los avances en el cuidado de estos pacientes. La naturaleza ubicua de los hongos facilita la colonización e infección por estos microrganismos. Si bien las bacterias continúan ocupando el primer lugar como agente causal (70\%), los hongos son los responsables del $20-25 \%$ de las infecciones ${ }^{12}$. Candida spp es el principal agente fúngico aislado de muestras de pacientes quemados y los mohos ocupan un lugar variable dentro de la epidemiología. 
Las principales características de los pacientes en los que hubo desarrollo de hongos filamentosos fueron las mismas que las publicadas por otros autores: grandes quemados, con períodos de internación prolongados (más de 10 días); aunque no se halló relación con la edad y la aparición de las lesiones ${ }^{10,13-15}$. Entre los pacientes ingresados en nuestro centro de quemados, el 17\% tuvo uno o más cultivos positivos para mohos con un examen microscópico positivo en el $93 \%$ de los casos, confirmando la infección y no colonización de las lesiones ${ }^{13}$. Este porcentaje es ampliamente superior a lo publicado en estudios realizados en diferentes centros alrededor del mundo, en los cuales, la prevalencia de infección por hongos filamentosos en este tipo de pacientes va desde el $0,04 \%$ en Australia ${ }^{3}, 1,4 \%$ en Estados Unidos ${ }^{1}, 1,7 \%$ en Francia $^{8}, 5,6 \%$ en India ${ }^{2}$. En Argentina, los registros provienen mayoritariamente de la población pediátrica, con una prevalencia del $12,8 \%{ }^{5}$. Esto debería llevar a que en nuestro medio se deba tener una mayor sospecha de etiología fúngica en caso de signos de infección en los tejidos.

En nuestro centro se halló que más del $50 \%$ de los hongos recuperados en cultivo eran dematiáceos. La diferencia se mantiene aún con el estudio de Rosanova, también realizado en Argentina, donde el 9,5\% (2/21) de las infecciones fueron causadas por hongos negros. En la mayoría de los estudios realizados en otros países, los hialohifomicetes constituyen los principales microrganismos hallados en los cultivos de pacientes quemados, siendo Aspergillus spp el género más frecuente, seguido por Fusarium spp. Los mucorales parecen tener baja prevalencia en este grupo de pacientes ${ }^{2-3,5,8,16}$. Respecto a los hongos negros, su prevalencia es menor a los géneros antes mencionados: 6,5\% en el trabajo de Katz, en asociación con especies de Aspergillus spp (Schofield y col) y sin registro en el estudios de Schaal ${ }^{16}$.

El rol que cumple la flora fúngica del ambiente hospitalario en las infecciones de pacientes quemados aún no está del todo claro ya que no todas las especies halladas en los estudios de control ambiental son recuperadas de las heridas de los pacientes, sin embargo, el estudio realizado por Chakrabarti y col. ${ }^{17}$ enfatiza la necesidad de descontaminar el ambiente de las unidades de quemados y subraya el potencial patogénico de estos microorganismos en pacientes inmunocomprometidos.

La mortalidad es otro factor que varía ampliamente entre estos pacientes así como los géneros fúngicos implicados. En nuestro centro, el $24 \%(n=7)$ fallecieron y Fusarium spp fue hallado en el $50 \%$ de los casos. En el estudio de Katz ${ }^{9}$, registraron un 25\% (3/12) de óbitos y hubo desarrollo de Aspergillus spp y Mucor spp. Schaal y col. ${ }^{8}$ obtuvieron datos similares (25\% de mortalidad; $6 / 20$ aspergilosis y $2 / 9$ mucormicosis). Estos valores son muy diferentes a los hallados por Schofield y col. ${ }^{16}$ quienes registraron un $100 \%$ de mortalidad en los pacientes con infecciones por especies de Mucor y Alternaria y un $90 \%$ debido a Fusarium spp. Ninguno de los estudios pudo atribuir estas muertes directamente a la infección por mohos, aunque el estudio realizado por Horvath y col. demostró que los pacientes que presentaban infección (no colonización) por hongos en sus heridas, tenían 8 veces más probabilidades de morir.

La implicancia de estas infecciones en el desenlace de los pacientes es incierta, ya que en ningún caso hubo exámenes pos mortem. Solo en un paciente, la causa de muerte fue registrada como falla multiorgánica secundaria a sepsis por Aspergillus spp. Según el estudio de Murray y col. ${ }^{18}$ en el $14 \%$ de las autopsias realizadas en quemados (14/97), la presencia de hongos filamentosos fue un factor importante en la mortalidad, con las heridas cutáneas como principal sitio de infección.

La causa de muerte se debió en todos los casos a shock cardiogénico irreversible con falla multiorgánica (FMO), lo cual coincide con el estudio realizado por Krishnan y col. ${ }^{14}$, siendo la sepsis su principal disparador.

El 76\% de los pacientes recibió tratamiento luego del diagnóstico microbiológico directo. No hubo diferencia significativa en la mortalidad entre los pacientes tratados y no tratados con antifúngico, pero esto podría deberse al limitado número de casos que no permite hacer inferencias estadísticas fiables.

Limitaciones: Debido a que el número de casos estudiados es bajo, es difícil definir con certeza los factores de riesgo asociados a los hongos filamentosos y así obtener datos que sean estadísticamente significativos, aunque la superficie corporal afectada y la internación prolongada fueron un factor común en todos los estudios citados. 


\section{Conclusiones}

Las infecciones por hongos filamentosos en pacientes internados en la UTIQ de nuestro hospital son más frecuentes respecto a lo reportado por otros países ${ }^{1-3,12-13,18}$.

Los hongos dematiáceos fueron los agentes fúngicos aislados con mayor frecuencia en el grupo de pacientes estudiados.

Conocer en cada centro de quemados la prevalencia y las especies de hongos halladas con mayor frecuencia, permitirá la elección del tratamiento empírico más adecuado.

\section{Bibliografía}

1. Ballard J, Edelman L, Saffle J, Sheridan R, Kagan R, Bracco D, et all. Positive fungal cultures in burn patients: a multicenter review. J Burn Care Res. 2008; 29: 213-21.

2. Capoor MR, Sarabahi S, Tiwari VK, Narayanan RP. Fungal infections in burns: Diagnosis and management. Indian $J$ Plast Surg. 2010;43 (Suppl S1):37-42

3. Katz T, Wasiak J, Cleland H, Padiglione A. Incidence of non-candidal fungal infections in severe burn injury: An Australian perspective. Burns: 40 (5): $881-886$.

4. Morales C, Gómez A, Ovidio J, Gallego M, Usuga Y, Hoyos M, et al. Infección en pacientes quemados del Hospital Universitario San Vicente de Paúl, Medellín Colombia.Rev. Colomb Cir. 2010; 25: 267-275.

5. Rosanova MT, Basilico H, Villasboas M, Finquelievich J, Mónaco A, Pérez G, et al. Infecciones fúngicas en una unidad de quemados pediátrica. Arch Argent Pediatr. 2011; 109:437-44

6. Struck MF, Gille J. Fungal infections in burns: a comprehensive review. Annals of burns and fire disasters. 2013; 26(3): 147.

7. Fayazov AD, Shukurov SI, Shukurov BI, Sultanov BC, Namazov AN, Ruzimuratov DA. Disorders of the Immune System in Severely Burned Patients. Annals of burns and fire disasters. 2009; 22(3):121-130.

8. Schaal JV, Leclerc T, Soler C, Donat N, Cirrode A, Jault P, Bargues L. Epidemiology of filamentous fungal infections in burned patients: A French retrospective study. Burns. 2015; 41 (4): $853-863$.

9. Murray CK, Loo FL, Hospenthal DR, Cancio LC, Jones JA, Kim SH, et al. Incidence of systemic fungal infection and related mortality following severe burns. Burns. 2008; 34 (8): 1108 - 1112.

10. Ezpeleta-Baquedano C, Barrios-Andrés JL, Delgado-Iribarren García Campero. A. Control microbiológico ambiental. Enferm Infecc Microbiol Clin. 2013; 31:396-401.

11. Rosanova MT, Stamboulian D, Lede R. Infecciones en los niños quemados: análisis epidemiológico y de los factores de riesgo. Arch Argent Pediatr. 2013; 111(4): 303-308.

12. Capoor MR, Gupta S, Sarabahi S, Mishra A, Tiwari VK, Aggarwal P. Epidemiological and clinico-mycological profile of fungal wound infection from largest burn centre in Asia. Mycoses. 2012; 55:181-8.

13. Horvath EE, Murray CK, Vaughan GM, Chung KK, Hospenthal DR, Wade CE, et al. Fungal wound infection (not colonization) is independently associated with mortality in burn patients. Ann Surg 2007; 245:978-85.

14. Krishnan P, Frew Q, Green A, Martin R, Dziewulski P. Cause of death and correlation with autopsy findings in burns patients Burns. 2012; 39 (4):583 - 588.

15. Sarabahi S, Tiwari VK, Arora S, Capoor MR, Pandey A. Changing pattern of fungal infection in burn patients. Burns. 2012; 38 (4): $520-528$.

16. Schofield CM, Murray CK, Horvath EE, Cancio LC, Kim SH, Wolf SE. Correlation of culture with histopathology in fungal burn wound colonization and infection. Burns 2007; 33:341-6.

17. Chakrabarti A, Chatterjee SS, Rao KL, Zameer MM, Shivaprakash, MR, Singhi S. Recent experience with fungemia: Change in species distribution and azole resistance. Scand J InfectDis. 2009; 41:275-84.

18. Murray CK, Loo FL, Hospenthal DR, Cancio LC, Jones JA, Kim SH, et al. Incidence of systemic fungal infection and related mortality following severe burns. Burns. 2008; 34 (8): $1108-1112$. 